\title{
Ansiedade na fase perinatal: revisão de literatura
}

\author{
Anxiety in the perinatal stage: literature review \\ Ansiedad en la etapa perinatal: revisión de la literatura
}

Recebido: 18/11/2021 | Revisado: 24/11/2021 | Aceito: 24/11/2021 | Publicado: 26/11/2021

Antônio Levi Sampaio de Araújo ORCID: https://orcid.org/0000-0002-8880-0736 Faculdade Santa Maria, Brasil E-mail: lev_sampaioaa@outlook.com

João Emmanuel Cardoso Andrade Xavier ORCID: https://orcid.org/0000-0001-7601-1636 Centro Universitário Unileão, Brasil E-mail: joaoemmanuel284@gmail.com

Maria Isadora Fernandes Lima ORCID: https://orcid.org/0000-0001-8452-7093 Faculdade Santa Maria, Brasil

E-mail: fernandeslima.isadora@gmail.com Adjane Pereira Jacó ORCID: https://orcid.org/0000-0001-7213-7434 Faculdade Santa Maria, Brasil E-mail: adj.medic@gmail.com

Maria Thais Caldas Araújo ORCID: https://orcid.org/0000-0002-4661-9760 Faculdade Santa Maria, Brasil E-mail: thaiscaldascalu@gmail.com

Paula Ívina Oliveira Silva Santos ORCID: https://orcid.org/0000-0003-0312-3463 Faculdade Santa Maria, Brasil E-mail: paulaiviina@ hotmail.com

Luiza Elias Morato

ORCID: https://orcid.org/0000-0002-5718-327X

Faculdade de Medicina de Barbacena, Brasil

E-mail: morato_luiza@hotmail.com

Maria Augusta Soares Sobreira Machado ORCID: https://orcid.org/0000-0002-6374-4432 Faculdade Santa Maria, Brasil

E-mail: mariaaugustamedicina@gmail.com

João Marcos Batista Gomes de Araujo ORCID: https://orcid.org/0000-0001-6877-2179 Faculdade Santa Maria, Brasil

E-mail: joaomarcosbg@ hotmail.com

Leandro Nonato da Silva Santos ORCID: https://orcid.org/0000-0003-3295-0409 Universidade Federal de Campina Grande, Brasil E-mail: leandrononato92@hotmail.com

\begin{abstract}
Resumo
A ansiedade é conceituada por um estado psíquico de apreensão ou um medo exacerbado, instigado pela antecipação de uma situação desagradável, perigosa ou até mesmo em uma situação desafiadora. O transtorno de ansiedade está presente no desenvolvimento humano, presente em todos os períodos da vida. No entanto, existem circunstancias que a ansiedade pode ser ativada, como o nascimento de um filho, causando adulterações psicológicas, podendo derivar complicações obstétricas, como o nascimento prematuro e baixo peso do bebê, pré-eclâmpsia e mecônio no líquido amniótico maior atividade motora no feto e depressão no período gestacional. Sendo assim, o presente trabalho tem como objetivo realizar uma estudo exploratório e de revisão de literatura sobre os transtornos de ansiedade que envolve a fase perinatal. A partir da literatura, verificou-se que a fase perinatal é um período vulnerável durante o qual ocorrem depressão e ansiedade. Isso acontece devido a uma combinação única de stressores neuro-hormonais e psicossociais, intensificando a vulnerabilidade das mulheres para sintomas depressivos e transtornos de ansiedade. Verificou-se que os sintomas de ansiedade relacionada à gravidez são análogos aos definidos para os transtornos de ansiedade. Contudo, as consequências comportamentais da ansiedade relacionada à gravidez semelham se aplicar exclusivamente a determinadas mulheres e podem servir como indicadores importantes da gravidade da doença.
\end{abstract}

Palavras-chave: Saúde da mulher; Transtornos de ansiedade; Gestação; Pós-parto. 


\begin{abstract}
Anxiety is conceptualized as a psychic state of apprehension or exacerbated fear instigated by anticipation of an unpleasant, dangerous or even challenging situation. The anxiety disorder is present in human development, present in all periods of life. However, there are circumstances that anxiety can be activated, such as the birth of a child, causing psychological changes, which may lead to obstetric complications, such as premature birth and low birth weight of the baby, pre-eclampsia and meconium in the amniotic fluid, increased motor activity in the fetus and depression during pregnancy. Thus, the present study aims to perform an exploratory study and literature review on anxiety disorders involving the perinatal phase. From the literature, it was found that the perinatal phase is a vulnerable period during which depression and anxiety occur. This is due to a unique combination of neurohormonal and psychosocial stressors, intensifying women's vulnerability to depressive symptoms and anxiety disorders. Pregnancy-related anxiety symptoms have been found to be analogous to those defined for anxiety disorders. However, the behavioral consequences of pregnancy-related anxiety similarly apply uniquely to certain women and may serve as important indicators of the severity of the disorder.
\end{abstract}

Keywords: Women's health; Anxiety disorders; Pregnancy; Postpartum.

\title{
Resumen
}

La ansiedad se conceptualiza como un estado psíquico de aprensión o miedo exacerbado, instigado por la anticipación de una situación desagradable, peligrosa o incluso desafiante. El trastorno de ansiedad está presente en el desarrollo humano, presente en todos los períodos de la vida. Sin embargo, existen circunstancias en las que se puede activar la ansiedad, como el nacimiento de un niño, provocando una adulteración psicológica, que puede derivar en complicaciones obstétricas, como parto prematuro y bajo peso del bebé, preeclampsia y meconio en el líquido amniótico, mayor actividad motora en el feto y depresión en el período gestacional. Por tanto, el presente trabajo tiene como objetivo realizar un estudio exploratorio y revisión de la literatura sobre los trastornos de ansiedad que involucran la fase perinatal. A partir de la literatura, se encontró que la fase perinatal es un período vulnerable durante el cual ocurren la depresión y la ansiedad. Esto se debe a una combinación única de estresores neurohormonales y psicosociales, que intensifica la vulnerabilidad de las mujeres a los síntomas depresivos y los trastornos de ansiedad. Se encontró que los síntomas de ansiedad relacionados con el embarazo son análogos a los definidos para los trastornos de ansiedad. Sin embargo, las consecuencias conductuales de la ansiedad relacionada con el embarazo parecen aplicarse exclusivamente a determinadas mujeres y pueden servir como indicadores importantes de la gravedad de la enfermedad.

Palabras clave: Salud de la mujer; Desórdenes de ansiedad; Gestación; Post parto.

\section{Introdução}

"A ansiedade é uma condição afetiva normal que, quando em excesso, pode acarretar distúrbios do humor, bem como de pensamento, de comportamento e da atividade fisiológica” (Almeida, Behlau, \& Leite, 2011, p. 384). A ansiedade é definida por um estado psíquico de apreensão ou um medo exacerbado, instigado pela antecipação de uma situação desagradável, perigosa ou até mesmo em uma situação desafiadora. Os transtornos de ansiedade são comuns e resultam em grandes sofrimentos e importante comprometimento funcional (Cury, 2017).

Para Chemello, Levandowski e Donelli (2017) é uma vivência comum, potencialmente típica e experimentada e se caracteriza por um sentimento difuso, desagradável e vago de apreensão, como inquietação, desconforto e taquicardia. Ou seja, é uma resposta a uma ameaça desconhecida, interna, vaga ou de origem conflituosa. Do mesmo modo, essa vivência faz parte das experiências humanas e é um estado emocional esperado e necessário para a sobrevivência, visto que na sua ausência, o ser humano não lutaria, não procuraria vencer obstáculos e nem fugiria de situações perigosas.

O transtorno de ansiedade está presente no desenvolvimento humano, presente em todos os períodos da vida. No entanto, existem circunstancias que a ansiedade pode ser ativada e, dependendo do momento, é considerada como um estado emocional esperado, como a espera do resultado de uma seleção de emprego exame médico, união entre pessoas, o nascimento de um filho, entre outros (Tuma \& Maser, 2019). A depressão e ansiedade têm manifestações distintas, porém, possuem fundamentos corriqueiros, que são síndromes heterogêneas, de maneira suposta conexas devido a características cotidianas, são fenômenos separados, os quais podem alternar-se ao longo do tempo, são manifestações distintas, conceitual e empiricamente (Lopes \& Santos, 2018). 
O objeto de estudo do presente trabalho está relacionado a ansiedade que envolve a fase perinatal, desde que a mulher tem o desejo de engravidar, gestação, parto e puerpério. De acordo com Fallon et al. (2016), os sintomas de ansiedade nas fases perinatais são cada vez mais reconhecidos devido à sua alta prevalência e impacto. Bayrampour et al. (2016) afirmam que a ansiedade relacionada à gestação está mais associada aos resultados maternos e infantis do que a ansiedade geral e a depressão, podendo constituir um conceito distinto.

Diversas pesquisas mostram uma alta prevalência de doenças psiquiátricas em gestantes em mulheres (Meades \& Ayers, 2011). Além disso, existe uma grande quantidade de pesquisas sobre os resultados adversos da psicologia na maternidade. A ansiedade na gestação é uma forma comum de doença psiquiátrica, sendo reflexo da resposta ao estresse, que ocorre quando o bem-estar pessoal é ameaçado durante a gravidez (Yuksel, Akin, \& Durna, 2014).

Para Cameron, Giesbrecht e Tomfohr-Madsen (2021), a ansiedade é uma das adulterações psicológicas mais corriqueiras na gravidez, com índices superiores aos do puerpério, podendo derivar complicações obstétricas, como o nascimento prematuro e baixo peso do bebê, pré-eclâmpsia e mecônio no líquido amniótico maior atividade motora no feto e depressão no período gestacional.

A ansiedade nessa fase está conexa a menor tempo de aleitamento materno exclusivo, podendo trazer consequências negativas para a relação mãe/ bebê. Também pode incidir sobre o crescimento e causar atraso no desenvolvimento infantil. Quanto à saúde mental materna, a ansiedade gestacional associou-se com depressão gestacional e foi indicativo para ansiedade e depressão puerperal, deixando a mulher vulnerável ao aparecimento de perturbações emocionais (Schiavo, Rodrigues, \& Perosa, 2018).

Do ponto de vista psicológico, a gravidez na mulher é considerada um momento crítico em sua vida, visto que as adequações podem alterar seu equilíbrio emocional e gerar estados de tensão, ansiedade e/ou medo, estando esta última emoção presente em aproximadamente $20 \%$ das gestações. Recentemente, surgiram pesquisas em relação ao efeito que a presença desses estados emocionais na evolução da gravidez e o desenvolvimento do feto, como, por exemplo, a sensação de falta de controle e/ou dor excessiva durante o parto, o aumento da dificuldade para dilatação e o aumento de recém-nascidos prematuros e de baixo peso (Molina-Fernández et al., 2015).

Nesse contexto, o presente trabalho tem como objetivo realizar uma estudo exploratório e de revisão de literatura sobre os transtornos de ansiedade que envolve a fase perinatal, desde que a mulher tem o desejo de engravidar, gestação, parto, puerpério e primeiros anos de vida da criança.

\section{Metodologia}

\subsection{Tipo de pesquisa}

Conforme caracteriza Köche (2016), do ponto de vista da natureza, esse trabalho trata de uma pesquisa básica, também conhecida como pesquisa fundamental, focada em ampliar o conhecimento que temos do mundo e tudo o que o forma. Pela perspectiva de abordagem, é uma pesquisa qualitativa, ou seja, é desenvolvido conceitos, ideias e entendimentos através de padrões encontrados nos dados, ao invés de coletar dados para comprovar teorias, hipóteses e modelos preconcebidos (Cardano, 2017). Para Souza Martins (2004, p. 1), a pesquisa qualitativa é conceituada "como aquela que privilegia a análise de microprocessos, através do estudo das ações sociais individuais e grupais, realizando um exame intensivo dos dados, e caracterizada pela heterodoxia no momento da análise".

Analisando os objetivos essa pesquisa é exploratória, ou seja, a finalidade é proporcionar maior familiaridade com o problema, tornar-se explicito ou construir hipóteses com seu respeito ou causar aprimoramento do tema (Neto, 2017). Em relação aos procedimentos técnicos, é do tipo Revisão Bibliográfica, no qual proporciona uma síntese de conhecimento e a incorporação dos resultados de estudos significativos, fundamentado em diversas pesquisas já publicadas. 
De acordo com Gil (2010), o estudo bibliográfico é tido desde a concepção do projeto e organização de ideias as compreende etapas (formulação do problema, a escolha do tema a ser investigada, a elaboração do plano de trabalho, a formulação dos objetivos, identificação, localização e obtenção de fontes que sejam capazes de fornecer os dados adequados à pesquisa desejada, leitura do material obtido, análise e interpretação lógica dos dados e redação final do texto).

\subsection{Procedimentos metodológicos}

O método científico consiste em um conjunto de passos e ferramentas pelo qual o pesquisador direciona seu projeto de trabalho com critérios de caráter científico para alcançar dados que suportam ou não sua teoria inicial, em que possui liberdade de definir quais os melhores instrumentos vão utilizar para cada tipo de pesquisa com finalidade de alcançar resultados confiáveis e com possibilidades de serem generalizados para outros casos (Praça, 2015).

Para a realização desse estudo, a primeira etapa foi a organização do problema a ser pesquisado, para posteriormente avaliar e aplicar todo o máximo do material bibliográfico disponível, uma vez que o tema deve conter relevância tanto teórica como prática e proporcionar interesse de ser estudado.

Foram utilizados os descritores validados através dos Descritores em Ciências da Saúde (DECS), o vocabulário DECS contém a terminologia padrão em ciências da saúde, em português, espanhol e inglês. Esse vocabulário "[...] serve como linguagem única para indexação, recuperação e navegação em todas as fontes de informação em 37 países na América Latina e do Caribe" (Pereira \& Montero, 2012, p. 2).

Foi utilizado para a indexação e navegações nas fontes de informações da Biblioteca Virtual em Saúde, foram criados pelo Centro Latino Americano e do Caribe de Informações em Ciência e Saúde BIREME/OPAS/OMS, tudo com intuito de facilitar a indexação de publicações científicas. Desta forma, para análise desta pesquisa, foi utilizada a base do DECS de acordo com a Biblioteca Virtual em Saúde: "Ansiedade", "Fase Perinatal” e "Gestação".

A pesquisa foi realizada na base de dados da Biblioteca Virtual de Saúde (BVS) por representar esta plataforma uma dissociação de conhecimentos científicos que surge das preocupações dos órgãos públicos nacionais e internacionais por oferecer através da internet uma informação científica de qualidade garantida. Nesta plataforma estão indexadas também outras bibliotecas virtuais, tais como SciELO (Scientific Electronic Library Online), BIRENE (Biblioteca Regional de Medicina), BDENF (Base de dados de enfermagem), LILACS (Literatura Latino-Americana e do Caribe em Ciências da Saúde), MEDLINE (Literatura Internacional em Ciências da Saúde), Coleciona SUS (Coleção Nacional das Fontes de Informação do SUS), MS (Acervo da Biblioteca do Ministério da Saúde), SAÚDE LEGIS (Legislação Federal da Saúde), OPAS (Acervo da Biblioteca da Organização Pan-Am. Saúde), WHOLIS (Sistema de Informação da Biblioteca da OMS).

Para organizar as informações dos trabalhos selecionados da base dados, foi utilizada a leitura flutuante dos títulos e resumos dos trabalhos bem como os resultados apresentados. O principal critério de exclusão de artigos está relacionado ao tempo da publicação, visto que apenas obras mais recentes são bem vistas no meio cientifico.

Critérios de inclusão: estudos encontrados na base de dado escolhida, publicados em período indeterminado, utilizando os descritores já citados. Critérios de exclusão: artigo noticiosos, textos em resenhas, artigos não indexados, opiniões, editoriais ou manuais. Para organizar as informações dos trabalhos selecionados da base dados, será utilizada a leitura flutuante dos títulos e resumos dos trabalhos, bem como os resultados apresentados.

No que se refere a delimitação temporal, o presente trabalho realizará uma pesquisa sobre o tema nos últimos 10 anos. Já no que se refere os Critérios de exclusão: artigo noticiosos, textos em resenhas, artigos não indexados, opiniões, editoriais ou manuais. Os resultados desta pesquisa geraram um conjunto inicial de documentos, que filtraremos os resultados iniciais da pesquisa a partir da leitura do título e do resumo. Todos os artigos encontrados, relacionados ao tema, foram incluídos na 
análise, independentemente de ser o assunto principal do artigo ou apenas mencionado no resumo. A Figura 1 apresenta o esquema metodológico utilizado na presente pesquisa.

Figura 1: Esquema Metodológico.

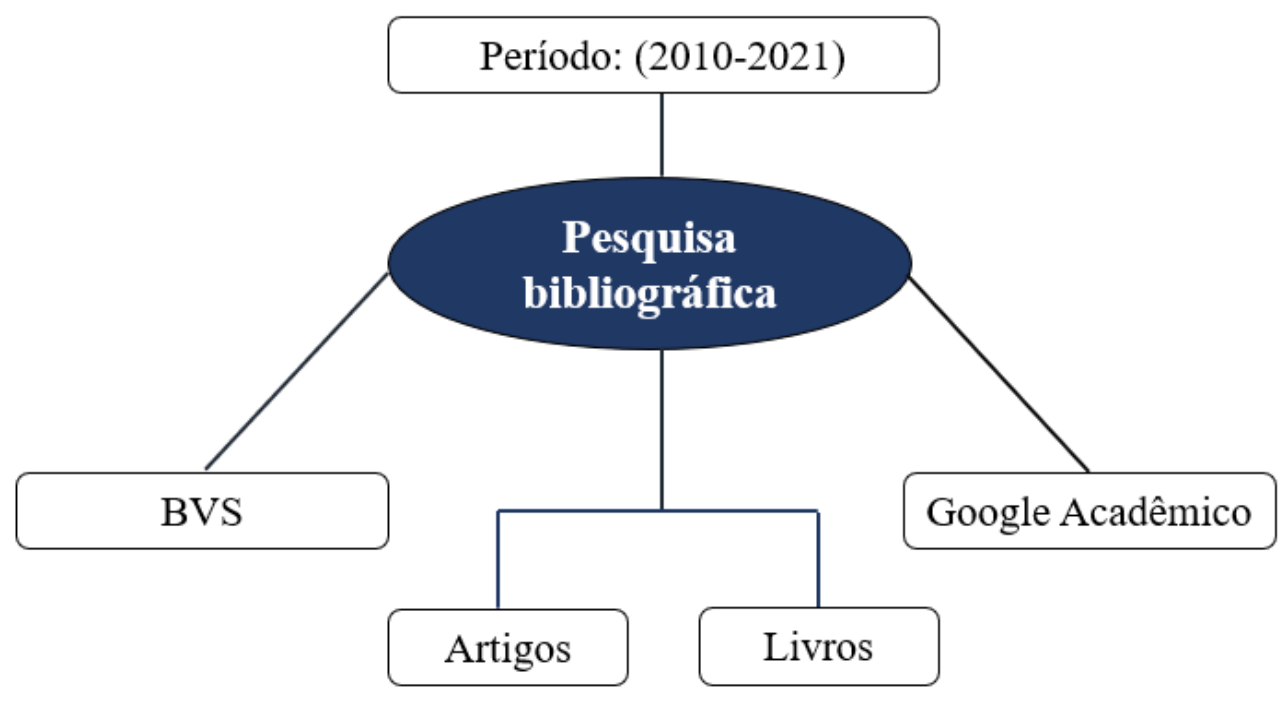

Fonte: Autores (2021).

Operacionalmente, foi adotada para a análise de dados a Análise Temática de Conteúdo, informações que segundo Minayo (2010), desdobra-se nas etapas pré-análise, exploração do material ou codificação e tratamento dos resultados obtidos/ interpretação. A etapa da pré-análise compreende a formulação e reformulação de hipóteses ou pressupostos, bem como a leitura flutuante e constituição do corpus. Durante a etapa da exploração do material, o investigador busca encontrar categorias que são expressões ou palavras significativas em função das quais o conteúdo de uma fala será organizado.

A categorização consiste num processo de redução do texto às palavras e expressões significativas. A partir daí, o analista propõe inferências e realiza interpretações, inter-relacionando-as com o quadro teórico desenhado inicialmente ou abre outras pistas em torno de novas dimensões teóricas e interpretativas, sugerida pela leitura do material (Minayo, 2010; Cavalcante, Calixto \& Pinheiro, 2014).

\section{Resultados e Discussões}

Os transtornos de ansiedade e os sintomas ansiosos têm crescido de forma significativa nos últimos anos, principalmente pelo modo de vida moderna, podendo ter consequências importantes na adaptação social, tendo em vista que modificam a qualidade das relações interpessoais, e podem afetar o exercício das atividades pessoais e profissionais (Beltrami, Moraes, \& Souza, 2013). Os autores ainda ressaltam que a chegada de uma criança alude alterações desde a gestação, tanto do ponto de vista fisiológico, quanto psíquico e sociofamiliar, visto que representa uma transição que faz parte da definição de um novo papel para a mulher.

Fairbrother et al. (2015) afirmam que o período perinatal é de particular importância, visto que o humor materno e as dificuldades de ansiedade estão associados a resultados adversos da gravidez, pais comprometidos, afeto e regulação do comportamento prejudicados e apego inseguro na prole. "De um lado, a gravidez, o parto e o puerpério representam períodos sensíveis no ciclo vital da mulher. Esses períodos envolvem grandes transformações, não só do ponto de vista fisiológico, mas também do ponto de vista psíquico e do papel sociofamiliar feminino" (Correia \& Linhares, 2007, p. 2). 
As mudanças físicas que ocorrem durante a gravidez podem provocar instabilidade emocional na mulher. A fase perinatal (gravidez e doze meses após o parto), devido a uma combinação única de stressores neuro-hormonais e psicossociais, intensifica a vulnerabilidade das mulheres para depressão e ansiedade. Estas são as perturbações mentais mais comuns no período perinatal e a sua prevalência e comorbilidade são ainda mais elevadas do que noutros períodos da vida da mulher (Xavier et al., 2019).

A fase perinatal é um período vulnerável durante o qual ocorrem depressão e ansiedade. Se não for tratada ou tratada de forma insuficiente, pode haver efeitos adversos significativos; portanto, o acesso a um tratamento rápido e eficaz é essencial (Hussain-Shamsy et al., 2020). O sofrimento perinatal foi amplamente conceituado como a experiência de depressão e / ou ansiedade. Pesquisas recentes mostraram que o estado afetivo de estresse também está presente durante o período perinatal, logo, pode colaborar para uma compreensão mais abrangente do sofrimento perinatal (Rallis et al., 2014).

Altieri et al. (2015) afirmam que nos Estados Unidos, aproximadamente 15\% das mulheres grávidas sofrem de transtornos de ansiedade e depressão, e grande parte recebem prescrição de antidepressivos. Como resultado, 5\% dos bebês nascidos nos Estados Unidos (200.000 / ano) são expostos via transmissão materna a inibidores seletivos da recaptação da serotonina (ISRS), logo, em estudos foram detectados efeitos de abstinência e atraso na coordenação motora em bebês expostos a ISRSs no útero.

Um estudo com objetivo de esclarecer o conceito de ansiedade relacionada à gravidez e identificar suas características e dimensões, bem como analisar os itens das medidas atuais de ansiedade relacionada à gravidez para determinar as dimensões e atributos que cada escala aborda, Bayrampour et al. (2016) concluíram as características críticas da ansiedade relacionada à gravidez são análogas aos definidos para os transtornos de ansiedade. Entretanto, as consequências comportamentais da ansiedade relacionada à gravidez semelham se aplicar exclusivamente a determinadas mulheres e podem servir como indicadores importantes da gravidade da doença.

Em uma pesquisa realizada por George et al. (2013), os autores identificaram que no pré-natal, 18,8\% das gestantes apresentavam sintomas graves de ansiedade, enquanto 20,2\% das mulheres apresentavam sintomas graves de ansiedade após o parto. Geralmente, as gestantes ansiosas utilizaram respostas de enfrentamento significativamente mais inadequadas e menos adaptativas, como autoculpa e negação da realidade, que permaneceram associadas à ansiedade no período perinatal. Os resultados desse estudo indicaram uma diminuição no enfrentamento adaptativo em mulheres sem ansiedade após o nascimento (por exemplo, aceitação, reenquadramento positivo). Os sintomas de ansiedade pré e pós-natal ocorrem com frequência e que o enfrentamento inadequado e / ou não funcional pode ser responsável pela persistência da ansiedade após o parto.

As análises de regressão múltipla, Prescott, Mackie e Rathbone (2018) mostraram que a ansiedade com a saúde aumentou durante a gravidez quando complicações médicas ocorreram em uma gravidez anterior e se sob tratamento médico para uma condição não relacionada à gravidez.

Rallis et al. (2014) em um estudo com objetivo de investigar as mudanças nos sintomas de depressão, ansiedade e estresse durante a gravidez e explorar as relações prospectivas entre esses sintomas, concluíram que os níveis de sintomas de depressão, ansiedade e estresse variam ao longo da gravidez. O aumento da depressão no início da gravidez parecia ser particularmente pertinente, visto que não só previa sintomas de depressão posteriores, mas também aumentava a ansiedade e o estresse no final da gravidez. Coletivamente, esses resultados destacam ainda mais a importância da triagem de saúde emocional no início da gravidez.

Além disso, estudos apontam que a ansiedade gestacional ocasiona implicações impresumíveis para o crescimento e desenvolvimento infantil, como também as alterações de humor que se refletem nas interações iniciais podem ter repercussões na protoconversação inicial entre mãe e bebê e, como consequência, no processo de aquisição da linguagem (Beltrami; 
Moraes, \& Souza, 2013). De acordo com Micali, Simonoff e Tesouro (2011), a prevalência da ansiedade generalizada é mais elevada em puérperas do que em mulheres da população geral, sendo mais comum que os transtornos depressivos.

Além das implicações já citadas anteriormente, Chemello, Levandowski e Donelli (2017) corroboram ao afirmar que no período puerperal a ansiedade pode influenciar a relação mãe-bebê, ocasionando repercussões para o desenvolvimento da criança. A ansiedade materna traria dificuldades para a relação mãe-bebê, visto que uma mãe ansiosa pode ter dificuldades em compreender as demandas do recém-nascido, decodificar o seu choro e as suas necessidades de amamentação, sono e afeto, o que pode comprometer o bem-estar do bebê.

Misri et al. (2015) identificaram que o transtorno de ansiedade generalizada perinatal (TAG) tem uma alta prevalência de $8,5 \%-10,5 \%$ durante a gravidez e $4,4 \%-10,8 \%$ no pós-parto. De acordo com os autores, embora de sua disfunção concomitante no paciente, essa condição de saúde mental potencialmente debilitante é frequentemente subdiagnosticada.

\section{Considerações finais}

O presente estudo buscou realizar uma estudo exploratório e de revisão de literatura sobre os transtornos de ansiedade que envolve a fase perinatal, desde que a mulher tem o desejo de engravidar, gestação, parto, puerpério e primeiros anos de vida da criança.

A partir da literatura, verificou-se que a fase perinatal é um período vulnerável durante o qual ocorrem depressão e ansiedade. Isso acontece devido a uma combinação única de stressores neuro-hormonais e psicossociais, intensificando a vulnerabilidade das mulheres para sintomas depressivos e transtornos de ansiedade. Verificou-se que os sintomas de ansiedade relacionada à gravidez são análogos aos definidos para os transtornos de ansiedade. Contudo, as consequências comportamentais da ansiedade relacionada à gravidez semelham se aplicar exclusivamente a determinadas mulheres e podem servir como indicadores importantes da gravidade da doença.

Sendo assim, recomenda-se para futuros trabalhos uma pesquisa sobre atuação dos profissionais de saúde na depressão pós-parto. Espera-se que o presente trabalho corrobore com a temática em questão e sirva de base para futuras pesquisas.

\section{Referências}

Almeida, A. A. Figueirêdo de., Behlau, M., \& Leite, J. R. (2011). Correlação entre ansiedade e performance comunicativa. Revista da Sociedade Brasileira de Fonoaudiologia, 16, 384-389.

Altieri, S. C. et al. (2015). Programação perinatal vs programação genética de estados de serotonina associados à ansiedade. Neuropsicofarmacologia, 40(6), 1456-1470.

Bayrampour, H. et al. (2016). Ansiedade relacionada à gravidez: uma análise de conceito. Revista Internacional de Estudos de Enfermagem, 55, 115-130.

Beltrami, L., De Moraes, A. B., \& De Souza, A. P. R. (2013). Ansiedade materna puerperal e risco para o desenvolvimento infantil. Distúrbios da Comunicação, 25(2).

Cameron, E. E., Giesbrecht, G. F., \& Tomfohr-Madsen, L. M. (2021). Propriedades psicométricas da Escala de Ansiedade Relacionada à Gravidez para uso com pais durante a gravidez. Psicologia dos Homens e Masculinidades, 22(1), 26.

Cardano, M. (2017). Manual de pesquisa qualitativa. A contribuição da teoria da argumentação. Tradução: Elisabeth da Rosa Conill. Vozes.

Cavalcante, R. B., Calixto, P., \& Pinheiro, M. M. K. (2014). Análise de conteúdo: considerações gerais, relações com a pergunta de pesquisa, possibilidades e limitações do método. Informação \& Sociedade: Estudos, 24(1), 13-18.

Correia, L. L., \& Linhares, M. B. M. (2007). Ansiedade materna nos períodos pré e pós-natal: revisão da literatura. Revista Latino-Americana de Enfermagem, $15,677-683$.

Cury, A. J. (2017). Ansiedade: como enfrentar o mal do século. Saraiva Educação SA. 
Research, Society and Development, v. 10, n. 15, e566101523521, 2021

(CC BY 4.0) | ISSN 2525-3409 | DOI: http://dx.doi.org/10.33448/rsd-v10i15.23521

Donelli, T. M. S., C., M. R., \& Levandowski, D. C. (2017). Ansiedade materna e maternidade: Revisão crítica da literatura. Interação em Psicologia, 21(1).

Fairbrother, N. et al. (2015). Depressão e ansiedade durante o período perinatal. BMC psiquiatria, 15(1), 1-9.

Fallon, V et al. (2016). A escala de ansiedade específica pós-parto: desenvolvimento e validação preliminar. Arquivos da saúde mental da mulher, 19(6), 10791090 .

George, A. et al. (2013). Sintomas de ansiedade e estratégias de enfrentamento no período perinatal. Gravidez e parto BMC, 13(1), 1-6.

Gil, A. C. et al. (2002). Como elaborar projetos de pesquisa. Atlas.

Hussain-Shamsy, Neesha et al. (2020). Saúde móvel para depressão e ansiedade perinatal: revisão do escopo. Journal of medical Internet research, 22(4), e17011.

Köche, J. C. (2016). Fundamentos de metodologia científica. Editora Vozes.

Lopes, K. C. da S. P., \& Dos Santos, W. L. (2018). Transtorno de ansiedade. Revista de Iniciação Científica e Extensão, 1(1), 45-50.

Meades, R., \& Ayers, Susan. (2011). Medidas de ansiedade validadas em populações perinatais: uma revisão sistemática. Jornal de transtornos afetivos, $133(1-2), 1-15$.

Micali, N., Simonoff, E., \& Tesouro, Janet. (2011). Gravidez e depressão pós-parto e ansiedade em uma coorte longitudinal da população geral: o efeito dos transtornos alimentares e depressão passada. Jornal de transtornos afetivos, 131(1-3), 150-157.

Minayo, M. C. S. (2010). Introdução. In: Minayo, M. C. S.; Assis, S. G.; Souza, E. R. (Org.). Avaliação por triangulação de métodos: Abordagem de Programas Sociais. Fiocruz, p. 19-51.

Misri, S. et al. (2015). Transtorno de ansiedade generalizada perinatal: avaliação e tratamento. Jornal da saúde da mulher, 24(9), 762-770.

Molina-Fernández, Inmaculada de et al. (2015). Ansiedade e medos das gestantes diante do parto: A importância da sua detecção. Revista portuguesa de enfermagem de saúde mental, (13), 17-24.

Neto, J. A. M. (2017). Metodologia científica na era da informática. Saraiva Educação AS.

Pereira, T. A., \& Montero, E. F. de S. (2012). Terminologia DeCS e as novas regras ortográficas da língua portuguesa: orientações para uma atualização. Acta Cirúrgica Brasileira, 27(7), 509-514.

Praça, F. S. G. (2015). Metodologia da pesquisa científica: organização estrutural e os desafios para redigir o trabalho de conclusão. Revista Eletrônica "Diálogos Acadêmicos, 8(1), 72-87.

Prescott, J., Mackie, L., \& Rathbone, A. L. (2018). Preditores de ansiedade em relação à saúde durante a gravidez. Mhealth, 4.

Rallis, S. et al. (2014). Um exame prospectivo de depressão, ansiedade e estresse durante a gravidez. Mulheres e Nascimento, 27(4), e36-e42.

Schiavo, R. de A., Rodrigues, O. M. P. R., \& Perosa, G. B. (2018). Variáveis associadas à ansiedade gestacional em primigestas e multigestas. Trends in Psychology, 26, 2091-2104.

Souza Martins, H. H. T. (2004). Metodologia qualitativa de pesquisa. Educação e pesquisa, 30(2), 289-300.

Tuma, A. Hussain; M., \& Jack D. (2019). (Ed.). Ansiedade e os transtornos de ansiedade. Routledge.

Xavier, J. B. (2019). Depressão pós-parto: atuação da enfermagem na prevenção. 2019. 49 f. Trabalho de Conclusão de Curso - Faculdade de Educação e Meio Ambiente - FAEMA.

Yuksel, F., Akin, S., \& Durna, Z. (2014). Sofrimento pré-natal em mulheres grávidas turcas e fatores associados ao sofrimento pré-natal materno. Jornal de enfermagem clínica, 23(1-2), 54-64. 